Publ. Mat. 58 (2014), 233-249

DOI: 10.5565 /PUBLMAT_58114_12

\title{
ON RINGS WITH FINITE NUMBER OF ORBITS
}

\author{
Mągorzata HryniewickA AND JAN KREMPA
}

\begin{abstract}
Let $R$ be an associative unital ring with the unit group $U(R)$. Let $\mathcal{S}$ denote one of the following sets: the set of elements of $R$, of left ideals of $R$, of principal left ideals of $R$, or of ideals of $R$. Then the group $U(R) \times U(R)$ acts on the set $\mathcal{S}$ by left and right multiplication. In this note we are going to discuss some properties of rings $R$ with a finite number of orbits under the action of $U(R) \times U(R)$ on $\mathcal{S}$.
\end{abstract}

2010 Mathematics Subject Classification: 16P99, 16U60, 16L30.

Key words: Groups of units, $U(R)$-orbits, semilocal rings, semiprimary rings.

\section{Preliminaries}

Throughout this note, by a ring we mean an associative ring with unit. All considered modules are assumed to be left. We adapt the terminology from [La91]. For a ring $R$, we denote by $R^{+}$the additive group of $R$. The unit group of $R$, the Jacobson radical of $R$ and the prime radical of $R$ are denoted by $U(R), J(R)$ and $P(R)$, respectively. In the note we concentrate on the natural group action of $U(R) \times U(R)$ on $R^{+}$defined by

$$
(a, b) \rightarrow x=a x b^{-1},
$$

for all $a, b \in U(R), x \in R$. The action (1.1) induces in a natural way an action of the group $U(R) \times U(R)$ on the set of left (respectively, principal left) ideals of $R$ and of ideals of $R$, however the action on the latter set is trivial. Orbits under the action (1.1) are called simply $U$-orbits, and belonging to the same $U$-orbit is called a $U$-equivalence. Motivated by results from [Hi04, OR03], for a ring $R$, we are going to consider the following properties:

FNE $\quad R$ has only a finite number of $U$-orbits of elements.

FNPLI $R$ has only a finite number of $U$-orbits of principal left ideals. FNLI $R$ has only a finite number of $U$-orbits of left ideals.

FNI $\quad R$ has only a finite number of $U$-orbits of ideals $(R$ has only a finite number of ideals). 
For a ring $R$, we can directly verify the following connections between the above properties:

$$
\mathrm{FNE} \Rightarrow \mathrm{FNPLI} \Rightarrow \mathrm{FNI} \text { and } \mathrm{FNLI} \Rightarrow \mathrm{FNPLI} \Rightarrow \mathrm{FNI} \text {. }
$$

Every property listed above is closed under taking homomorphic images and finite (but not infinite) direct products.

Since for any division ring $D$ and any positive integer $n$, the $n \times$ $n$ matrix ring $M_{n}(D)$ has exactly $n+1 U$-orbits both of elements and of left ideals (see [Ok98, Lemma 2.1]), it follows that $M_{n}(D)$ satisfies every property listed in Formula (1.2). Hence, we have:

Theorem 1.1. Every J-semisimple artinian ring satisfies all the properties listed in Formula (1.2).

On the other hand, we have the following well-known example.

Example 1.2. Let $\mathbb{K}$ be an infinite field, and let $R=\mathbb{K}[x, y] /\left(x^{2}, x y, y^{2}\right)$ be the homomorphic image of the polynomial ring in commuting variables $x, y$. Then $R$ is a 3 -dimensional $\mathbb{K}$-algebra. Since $R$ has an infinite number of ideals, it follows that $R$ satisfies none of the properties listed in Formula (1.2).

The question about an action of important subgroups of the group $U(R) \times U(R)$ on $R^{+}$is also of interest to us, because the finiteness of the set of orbits under this action implies that of the set of orbits under the action (1.1). For example, Yasuyuki Hirano proved the following:

Theorem 1.3 ([Hi04, Theorem 2.4]). For every ring $R$, the following conditions are equivalent:

1. $R$ has only a finite number of orbits under the action of the group $U(R) \times 1$ on $R^{+}$.

2. $R$ has only a finite number of left ideals.

If $R$ satisfies these equivalent conditions, $R$ is a left artinian ring. More precisely, $R$ is the direct sum of a finite ring and a finite number of principal left ideal left artinian rings.

In Example 3.4 we see that a ring satisfying the conditions of the above theorem need not be right artinian.

Motivated by Theorem 1.1, Example 1.2 and Theorem 1.3, we are going to discuss two questions. One of them is: Under which conditions does a left and/or right artinian ring satisfy FNE or a similar property? The other is: Must every ring satisfying FNE or a similar property be left and/or right artinian, or at least semiprimary? 


\section{Some partial results}

Every ring satisfying the conditions of Theorem 1.3 evidently also satisfies the properties listed in Formula (1.2). As an easy consequence of Theorem 1.3, [Jo79, Proposition 1] and [JKN88, Theorem 1.3] we obtain:

Theorem 2.1. For every commutative ring $R$, the following statements are equivalent:

1. $R$ satisfies all the properties listed in Formula (1.2).

2. $R$ satisfies any of the properties listed in Formula (1.2).

3. $R$ is the direct sum of a finite ring and a finite number of principal ideal local artinian rings.

4. $R$ has only a finite number of isomorphism classes of cyclic modules.

For arbitrary rings, FNI is the weakest among properties considered here. About rings satisfying this property we have:

Theorem 2.2. Assume a ring $R$ satisfies FNI. Then:

1. If $c \in R$ is a central regular element, then $c \in U(R)$.

2. $R=S \oplus T$, where $S$ is an algebra over the field $\mathbb{Q}$, and $m T=0$ for some positive integer $m$ (the case where $S=0$ or $T=0$ is possible).

3. $P(R)$ is nilpotent.

4. If $R$ is left or right noetherian, then $J(R)$ is nilpotent.

5. If every prime image of $R$ is simple artinian, then $R$ is semiprimary.

6. If $R$ satisfies a polynomial identity, then $R$ is semiprimary.

Proof: 1 . Let $c \in R$ be a central regular element. Consider the sequence of ideals $R c \supseteq R c^{2} \supseteq \cdots$ of $R$. From the assumption on a finite number of ideals of $R$ we see that $c^{n}=r c^{n+1}$ for some $n \geq 1$ and $r \in R$. The regularity of $c$ implies that $1=r c$, and so $c \in U(R)$.

2. Compare with [Fu73, §122]. For any ideal $I$ of $R$ and any positive integer $n$, we denote by $I_{[n]}$ and $n I$ the sets $\{x \in I \mid n x=0\}$ and $\{n x \mid x \in I\}$, respectively. Evidently, $I_{[n]}$ and $n I$ are ideals of $R$. Let $T$ denote the torsion part of $R^{+}$. Then $T=\bigcup_{n \geq 1} R_{[n]}=\sum_{n \geq 1} R_{[n]}$. The existence of only a finite number of ideals of $R$ implies that $T=R_{[m]}$ for any sufficiently large $m$, and so $m T=0$. On the other hand, for every $n \geq 1$, the sequence of ideals $n R \supseteq n^{2} R \supseteq \cdots$ stabilizes after a finite number of steps, say $n^{k} R=n^{k+1} R$. Therefore $n^{k}(R / T)=$ $n^{k+1}(R / T)$, and since $(R / T)^{+}$is a torsion-free group, it follows that 
$R / T=n(R / T)$, which means that $R / T$ is an algebra over the field $\mathbb{Q}$. Finally, let $S=m R$. Then $S \cap T=m R \cap R_{[m]}=0$. Moreover, since $R / T=m R / T=S / T$, hence $R=S+T$, and so $R=S \oplus T$.

The statement 3 follows immediately from the definition of the prime radical, while the statement 4 follows directly from Nakayama's Lemma.

5. Assume that every prime image of $R$ is simple artinian. Let $P_{1}$, $P_{2}, \ldots, P_{n}$ be all prime ideals of $R$. According to the above assumption, $P_{1}, P_{2}, \ldots, P_{n}$ are all maximal ideals of $R$. From this and the Chinese Remainder Theorem for rings it follows that

$$
R / P(R)=R /\left(P_{1} \cap P_{2} \cap \cdots \cap P_{n}\right) \cong R / P_{1} \times R / P_{2} \times \cdots \times R / P_{n}
$$

is a $J$-semisimple artinian ring, and hence $J(R)=P(R)$. From the statement 3 we see that $J(R)$ is a nilpotent ideal. The conclusion is now evident.

6 . Let $R$ be a prime PI-ring satisfying FNI. A direct calculation shows that every nonzero central element of $R$ is regular, and, in consequence, $R$ is a central prime PI-algebra over a field by the statement 1 . According to Rowen's Theorem and Kaplansky's Theorem, $R$ is a simple artinian ring.

Now, let $R$ be an arbitrary PI-ring satisfying FNI. From the previous case and the statement $5, R$ is a semiprimary ring.

In [OR03, p. 484], Jan Okniński and Lex E. Renner conjectured that every ring satisfying FNLI is semiprimary, and hence artinian. We are able to prove this only under some additional assumptions.

Theorem 2.3. Assume a ring $R$ satisfies FNLI. Then $R$ is semilocal. Moreover, $R$ is semiprimary provided at least one of the following conditions is fulfilled:

1. $J(R)$ is nil.

2. Every prime image of $R$ is left bounded (such a ring $R$ is called left fully bounded).

Theorem 2.4. Assume a ring $R$ satisfies FNPLI. Then $R$ is semiprimary provided at least one of the following conditions is fulfilled:

1. $R$ is semilocal and $J(R)$ is nil.

2. $R$ satisfies $A C C$ or DCC on principal left ideals.

In the proofs of the above theorems we use some auxiliary results, sometimes giving more information than we need to prove the above theorems. 
Lemma 2.5. Assume a ring $R$ satisfies FNPLI. Then every one-sided nil-ideal of $R$ is nilpotent of nilpotency index not greater than $n+1$, where $n$ denotes the number of $U$-orbits of principal left ideals of $R$. In particular, $R / P(R)$ contains no one-sided nil-ideals.

Proof: Let $I$ be a one-sided nil-ideal of $R$. Suppose that there exist elements $x_{1}, x_{2}, \ldots, x_{n+1}$ of $I$ with $x_{1} x_{2} \cdots x_{n+1} \neq 0$. Out of all the left ideals $R x_{1}, R x_{1} x_{2}, \ldots, R x_{1} x_{2} \cdots x_{n+1}$, at least two are $U$-equivalent to each other, say $R x_{1} x_{2} \cdots x_{i}$ and $R x_{1} x_{2} \cdots x_{i} x_{i+1} \cdots x_{j}$. Let $x=$ $x_{1} x_{2} \cdots x_{i}$ and $y=x_{i+1} \cdots x_{j}$. Then $x=r x y b^{-1}$ for some $r \in R$ and $b \in U(R)$. By induction on $m \geq 1$, we can check that $x=r^{m} x\left(y b^{-1}\right)^{m}$. Since either $y b^{-1} \in I$ or $b^{-1} y \in I$, hence, by assumption, $\left(y b^{-1}\right)^{m}=0$ for any sufficiently large $m$, which contradicts $x \neq 0$. This contradiction means that $I$ is nilpotent of nilpotency index not greater than $n+1$. Now, final conclusion is evident.

A version of the result below was noticed in [Me11, p. 10].

Lemma 2.6. If a ring $R$ has left ideals $R x \subsetneq R y$ belonging to the same $U$-orbit, then $R$ satisfies neither ACC nor DCC on principal left ideals.

Proof: By assumption, $R x b^{-1}=R y$ for some $b \in U(R)$. Then $R x \subsetneq$ $R x b^{-1}$. From this we conclude that

$$
\cdots \subsetneq R x b^{2} \subsetneq R x b \subsetneq R x \subsetneq R x b^{-1} \subsetneq R x b^{-2} \subsetneq \cdots
$$

is a strictly increasing sequence of principal left ideals of $R$, and the result follows.

Proof of Theorem 2.3: For any $U$-equivalent left ideals $I$ and $J$ of $R$, the quotient $R$-modules $R / I$ and $R / J$ are isomorphic. Considering intersections of finite sets of maximal left ideals of $R$ and applying the Jordan-Hölder Theorem to the quotient $R$-modules by these intersections we can check, as on p. 484 in [OR03], that $R$ is semilocal.

The statement 1 follows immediately from Lemma 2.5.

2. Let $R$ be a left bounded prime ring satisfying FNLI. Let $I$ be a minimal ideal of $R$, and let $0 \neq a \in I$. Let $M$ be a left ideal of $R$ maximal with respect to the property that $a \notin M$. Such a left ideal exists by Zorn's Lemma. Since in the $R$-module $R / M$ every nonzero submodule must contain $a+M$, it follows that $(M+R a) / M$ is a simple $R$-module. By assumption, $M$ is not an essential left ideal of $R$. From this $M+R a \subseteq M \oplus K$ for some nonzero left ideal $K$ of $R$, and hence $(M+R a) / M \subseteq(M \oplus K) / M \cong K$. This means that the left socle of $R$, 
say $\operatorname{soc}(R)$, is a nonzero ideal of $R$. However, $R$ satisfies FNLI. Therefore $\operatorname{soc}(R)$ is of finite length as an $R$-module, and thus $\operatorname{soc}(R)$ is a unital ring. By the primeness of $R$ we obtain that $R=\operatorname{soc}(R)$ is a simple artinian ring.

Now, let $R$ be an arbitrary left fully bounded ring satisfying FNLI. Then, by the above consideration, $R$ satisfies the assumptions of the statement 5 in Theorem 2.2, and hence is semiprimary.

Proof of Theorem 2.4: The statement 1 follows immediately from the assumption and Lemma 2.5.

2. Suppose that there exists a strictly decreasing sequence of principal left ideals $R x_{1} \supsetneq R x_{2} \supsetneq \cdots$ of $R$. Since, by assumption, $R$ satisfies FNPLI, there exist $m \neq n$ for which the left ideals $R x_{m}$ and $R x_{n}$ belong to the same $U$-orbit, and according to Lemma 2.6, $R$ does not satisfy ACC on principal left ideals, which contradicts the assumption. We proved thereby that $R$ satisfies DCC on principal left ideals. According to the Bass Theorem, $R$ is right perfect, that is, $R$ is semilocal and $J(R)$ is right T-nilpotent. In particular, $J(R)$ is nil (see [La91, §23] for more details). Now, we conclude from the statement 1 that $R$ is semiprimary.

In Example 3.3 we see that the property FNPLI does not imply the property FNLI, even for PI-rings. Example 3.7 shows that even if an algebra is finite dimensional over a field, the property FNPLI needs not imply the property FNLI. In Example 3.8 we see a finite dimensional algebra over a field satisfying FNI and simultaneously not satisfying FNPLI. According to [OR03, Lemma 1], under the assumption on semiperfectness of a ring, the property FNPLI implies the property FNE.

In Example 3.5 we see a non-artinian algebra over a field still satisfying FNPLI. Example 1.2 shows that in many cases the converse of the above theorems is not necessarily true. In many cases, applying the following result, we can obtain the left artinian condition on rings from the above theorems.

Theorem 2.7. Let $R$ be a semiprimary ring. Then $R$ is left artinian provided at least one of the following conditions is fulfilled:

1. $R$ satisfies FNLI.

2. $J(R)$ is a finitely generated $R$-module.

3. $R$ satisfies FNI and is a finitely generated PI-algebra over its center.

In particular, every ring satisfying both FNLI and ACC or DCC on principal left ideals is left artinian. 
Proof: Let $R$ be a semiprimary ring.

1. Let $R$ satisfies FNLI. By induction on the nilpotency index of $J(R)$, we can check that $R$ is left artinian.

2. If $J(R)$ is finitely generated as an $R$-module, then $R$ is left artinian by a theorem of Hopkins type.

The statement 3 follows immediately from [JKN88].

\section{Examples}

For a subfield $\mathbb{K}$ of a field $\mathbb{F}$, the field extension degree of $\mathbb{F}$ over $\mathbb{K}$ is denoted by $[\mathbb{F}: \mathbb{K}]$. We start with the following observation.

Lemma 3.1. Let $\mathbb{K}$ be an infinite subfield of a field $\mathbb{F}$ with $[\mathbb{F}: \mathbb{K}]=$ $n \leq \infty$. Consider the natural action of the group $U(\mathbb{F})$ on the set of all $\mathbb{K}$-vector subspaces of $\mathbb{F}$ by multiplication. Then:

1. All 1-dimensional $\mathbb{K}$-vector subspaces of $\mathbb{F}$ belong to the same $U$-orbit.

2. If $n=2$ or $n=3$, then all 2-dimensional $\mathbb{K}$-vector subspaces of $\mathbb{F}$ belong to the same $U$-orbit.

3. If $n \geq 4$, then the field $\mathbb{F}$ has an infinite number of $U$-orbits of 2-dimensional $\mathbb{K}$-vector subspaces.

Proof: The statement 1 is obvious because $\mathbb{F}$ is a field.

2. In the case when $n=2$, the claim is evident. For the case when $n=$ 3 , we fix an element $x \in \mathbb{F} \backslash \mathbb{K}$. Then the field $\mathbb{F}$ is a simple extension of the field $\mathbb{K}$ with a generator $x$. As a basis of this extension we can take $\left\{1, x, x^{2}\right\}$. From the choice of $x$ we have $x^{3}=a_{0}+a_{1} x+a_{2} x^{2}$ for some $a_{0}, a_{1}, a_{2} \in \mathbb{K}$. In the proof of this case we will consider the scalar product of $\mathbb{F}$ connected with this basis.

Let $V$ be a 2-dimensional $\mathbb{K}$-vector subspace of $\mathbb{F}$. Consider $V^{\perp}=$ $\operatorname{span}_{\mathbb{K}}(y)$, the subspace orthogonal to $V$. We claim that $\operatorname{span}_{\mathbb{K}}(y)^{\perp}=$ $\operatorname{span}_{\mathbb{K}}(b, b x)$ for some $b \in U(\mathbb{F})$. Indeed, set $y=y_{0}+y_{1} x+y_{2} x^{2}$ and $b=b_{0}+b_{1} x+b_{2} x^{2}$, where $y_{0}, y_{1}, \ldots, b_{2} \in \mathbb{K}$ and $b_{0}, b_{1}, b_{2}$ are searched for. Then $b x=a_{0} b_{2}+\left(b_{0}+a_{1} b_{2}\right) x+\left(b_{1}+a_{2} b_{2}\right) x^{2}$. The orthogonalities $y \perp b$ and $y \perp b x$ hold if and only if $y_{0} b_{0}+y_{1} b_{1}+y_{2} b_{2}=0$ and $y_{0} a_{0} b_{2}+y_{1}\left(b_{0}+\right.$ $\left.a_{1} b_{2}\right)+y_{2}\left(b_{1}+a_{2} b_{2}\right)=0$. We obtain the homogeneous system of two linear equations in three variables $b_{0}, b_{1}, b_{2}$ over the field $\mathbb{K}$

$$
y_{0} b_{0}+y_{1} b_{1}+y_{2} b_{2}=0 \text { and } y_{1} b_{0}+y_{2} b_{1}+\left(a_{0} y_{0}+a_{1} y_{1}+a_{2} y_{2}\right) b_{2}=0 \text {, }
$$


which has a nonzero solution. Thereby there exists an element $b \in U(\mathbb{F})$ such that $\operatorname{span}_{\mathbb{K}}(y)^{\perp}=\operatorname{span}_{\mathbb{K}}(b, b x)$ as we claimed. Obviously, $V=$ $\left(V^{\perp}\right)^{\perp}=\operatorname{span}_{\mathbb{K}}(y)^{\perp}=\operatorname{span}_{\mathbb{K}}(b, b x)$. This means that $V$ belongs to the $U$-orbit which the subspace $\operatorname{span}_{\mathbb{K}}(1, x)$ belongs to.

3. Now, assume that $n \geq 4$. We divide the consideration into three subcases.

3.I. There exists an element $x \in \mathbb{F} \backslash \mathbb{K}$ with $\mathbb{K}$-independent $1, x, x^{2}, x^{3}$. We let $V_{a}=\operatorname{span}_{\mathbb{K}}\left(1, x+a x^{2}\right)$ for every $a \in \mathbb{K}$. Both subspaces $V_{a}$ and $V_{b}$ belong to the same $U$-orbit if and only if $\operatorname{span}_{\mathbb{K}}\left(1, x+a x^{2}\right)=$ $\operatorname{span}_{\mathbb{K}}\left(c, c x+b c x^{2}\right)$ for some $c \in U(\mathbb{F})$. We will use that $a x-x^{2}, x^{3} \in$ $\operatorname{span}_{\mathbb{K}}\left(1, x+a x^{2}\right)^{\perp}=\operatorname{span}_{\mathbb{K}}\left(c, c x+b c x^{2}\right)^{\perp}$. Before this we set $c=$ $c_{0}+c_{1}\left(x+a x^{2}\right)=c_{0}+c_{1} x+a c_{1} x^{2}$, where $c_{0}, c_{1} \in \mathbb{K}$, not both 0 . Then $c x+b c x^{2}=c_{0} x+\left(b c_{0}+c_{1}\right) x^{2}+\left(a c_{1}+b c_{1}\right) x^{3}+a b c_{1} x^{4}$.

If $x^{4} \notin \operatorname{span}_{\mathbb{K}}\left(1, x, x^{2}, x^{3}\right)$, then the orthogonalities $\left(a x-x^{2}\right) \perp(c x+$ $\left.b c x^{2}\right)$ and $x^{3} \perp\left(c x+b c x^{2}\right)$ hold if and only if $a c_{0}-\left(b c_{0}+c_{1}\right)=0$ and $a c_{1}+b c_{1}=0$. We obtain the homogeneous system of two linear equations in two variables $c_{0}, c_{1}$ over the field $\mathbb{K}$

$$
(a-b) c_{0}-c_{1}=0 \text { and }(a+b) c_{1}=0,
$$

which has a nonzero solution if and only if $(a-b)(a+b)=0$.

If $x^{4} \in \operatorname{span}_{\mathbb{K}}\left(1, x, x^{2}, x^{3}\right)$, then $x^{4}=d_{0}+d_{1} x+d_{2} x^{2}+d_{3} x^{3}$ for some $d_{0}, d_{1}, d_{2}, d_{3} \in \mathbb{K}$, hence $c x+b c x^{2}=a b c_{1} d_{0}+\left(c_{0}+a b c_{1} d_{1}\right) x+\left(b c_{0}+\right.$ $\left.c_{1}+a b c_{1} d_{2}\right) x^{2}+\left(a c_{1}+b c_{1}+a b c_{1} d_{3}\right) x^{3}$. The orthogonalities $\left(a x-x^{2}\right) \perp$ $\left(c x+b c x^{2}\right)$ and $x^{3} \perp\left(c x+b c x^{2}\right)$ mean that $a\left(c_{0}+a b c_{1} d_{1}\right)-\left(b c_{0}+c_{1}+\right.$ $\left.a b c_{1} d_{2}\right)=0$ and $a c_{1}+b c_{1}+a b c_{1} d_{3}=0$. The homogeneous system of two linear equations in two variables $c_{0}, c_{1}$ over the field $\mathbb{K}$

$$
(a-b) c_{0}+\left(a^{2} b d_{1}-1-a b d_{2}\right) c_{1}=0 \text { and }\left(a+b+a b d_{3}\right) c_{1}=0,
$$

which we obtained, has a nonzero solution if and only if $(a-b)(a+b+$ $\left.a b d_{3}\right)=0$.

3.II. Every element of the field $\mathbb{F}$ is $\mathbb{K}$-algebraic of degree not greater than 3 , and there exists a separable element $x \in \mathbb{F} \backslash \mathbb{K}$. Then for any element $y \in \mathbb{F} \backslash \mathbb{K}(x), \mathbb{K}(x, y)$ is, by Abel's Theorem, a simple extension of $\mathbb{K}$ with a generator $z$ for some $z \in \mathbb{F}$. The $\mathbb{K}$-algebraic degree of $z$ is not smaller than 4 , contrary to the assumption.

3.III. Every element of the field $\mathbb{F}$ is $\mathbb{K}$-algebraic of degree not greater than 3 , and none of elements from $\mathbb{F} \backslash \mathbb{K}$ is separable. Then char $\mathbb{K}=$ $p>0$, and $\mathbb{F}$ is a radical extension of the field $\mathbb{K}$. From the assumption we see that $p=2$ or $p=3$, and every element $x \in \mathbb{F} \backslash \mathbb{K}$ is $\mathbb{K}$-algebraic of 
degree exactly $p$, or $x^{p} \in \mathbb{K}$ to be precise. We fix elements $x \in \mathbb{F} \backslash \mathbb{K}$ and $y \in \mathbb{F} \backslash \mathbb{K}(x)$. Then elements $x^{i} y^{j}$ are $\mathbb{K}$-independent for all $i, j \leq p-1$. We let $V_{a}=\operatorname{span}_{\mathbb{K}}(1, x+a y)$ for every $a \in \mathbb{K}$. Considerations similar to those used in the Subcase 3.I prove that both subspaces $V_{a}$ and $V_{b}$ belong to the same $U$-orbit as far as $(a-b)(a+b)=0$.

In every of these subcases, $U$-orbits of 2 -dimensional $\mathbb{K}$-vector subspaces of $\mathbb{F}$ contain no more than two subspaces considered above. The infinity of the field $\mathbb{K}$ implies an infinite number of $U$-orbits of 2-dimensional $\mathbb{K}$-vector subspaces of $\mathbb{F}$, and the proof is complete.

In [Hi04], Yasuyuki Hirano gave a number of properties of rings satisfying conditions of Theorem 1.3. The next result is a modification of observation from [Hi04, Example 2.9].

Lemma 3.2. Let $\sigma$ be a non-surjective endomorphism of an infinite field $\mathbb{F}$, let $\mathbb{K}=\sigma(\mathbb{F})$, and let $R=\mathbb{F}[y ; \sigma] /\left(y^{2}\right)$ be the homomorphic image of the skew polynomial ring in one variable $y$. Then:

1. $R$ has a unique proper left ideal, namely $R \bar{y}=\mathbb{F} \bar{y}$, where $\bar{y}$ denotes the image of $y$ in $R$. Therefore $R$ is a local ring with the nilpotent Jacobson radical $J(R)=\mathbb{F} \bar{y}$.

2. $R$ has exactly three $U$-orbits both of elements and of (principal) left ideals.

3. Every proper right ideal of $R$ is of the form $V \bar{y}$, where $V$ is a nonzero $\mathbb{K}$-vector subspace of $\mathbb{F} . V \bar{y}$ is a principal right ideal of $R$ if and only if $V$ is a 1 -dimensional $\mathbb{K}$-vector space. Therefore some of right ideals of $R$ are not principal.

4. The $U$-orbit of a right ideal $V \bar{y}$ of $R$ consists of all right ideals of $R$ of the form $f V \bar{y}$, where $f \in U(\mathbb{F})$.

5. $R$ has an infinite number of principal right ideals, and only three $U$-orbits of principal right ideals.

6. $R$ is a PI-ring.

Proof: Evidently, $R=\mathbb{F} \overline{1} \oplus \mathbb{F} \bar{y}$ is a 2-dimensional left vector space over the non-central field $\mathbb{F}$ with $\bar{y}^{2}=\overline{0}$ and $\bar{y} f=\sigma(f) \bar{y}$ for every $f \in \mathbb{F}$. We can immediately check that for all $f, g \in \mathbb{F}, f \overline{1}+g \bar{y} \in U(R)$ if and only if $f \neq 0$. The proof of the first five statements is now a direct calculation. For the last statement, it is enough to note that the ring $R$ satisfies $\left(x_{1} x_{2}-x_{2} x_{1}\right)^{2}=0$.

We now apply Lemmas 3.1 and 3.2 to obtain: 
Example 3.3. Let $\mathbb{L}$ be a field, let $\mathbb{F}=\mathbb{L}(x)$ be the field of rational functions in one variable $x$, and let $\sigma: \mathbb{F} \rightarrow \mathbb{F}$ be the $\mathbb{L}$-endomorphism defined by $\sigma(x)=x^{n}$ for some positive integer $n$. Then, under the notation of Lemma 3.2, the ring $R$ is both left and right artinian, the latter follows from $\mathbb{F}=\mathbb{K} \oplus \mathbb{K} x \oplus \cdots \oplus \mathbb{K} x^{n-1}$. Furthermore, $R$ has exactly three $U$-orbits both of elements and of (principal) left ideals. According to Lemma 3.1, we have:

1. If $n=2$, then $R$ has exactly four $U$-orbits of right ideals. Three of them are $U$-orbits of principal right ideals, whereas the fourth is the $U$-orbit of the Jacobson radical $J(R)=\mathbb{F} \bar{y}$ [Hi04, Example 2.9].

2. If $n=3$, then $R$ has exactly five $U$-orbits of right ideals. Three of them are $U$-orbits of principal right ideals, the fourth consists of all right ideals of $R$ of the form $V \bar{y}$, where $V$ is a 2-dimensional $\mathbb{K}$-vector subspace of $\mathbb{F}$, and the fifth is the $U$-orbit of the Jacobson radical $J(R)=\mathbb{F} \bar{y}$.

3. If $n \geq 4$, then $R$ has exactly three $U$-orbits of principal right ideals, and an infinite number of $U$-orbits of right ideals.

Example 3.4. Let $\mathbb{L}$ be a field, let $\mathbb{F}=\mathbb{L}\left(x_{1}, x_{2}, \ldots\right)$ be the field of rational functions in infinitely many variables $x_{1}, x_{2}, \ldots$, and let $\sigma: \mathbb{F} \rightarrow$ $\mathbb{F}$ be the $\mathbb{L}$-endomorphism defined by $\sigma\left(x_{i}\right)=x_{i}{ }^{2}$ for every $i \geq 1$. Then, under the notation of Lemma 3.2, the ring $R$ is left artinian, but not right artinian. Furthermore, $R$ has exactly three $U$-orbits of elements, of left ideals, and of principal right ideals. Finally, $R$ has an infinite number of $U$-orbits of right ideals because $\mathbb{F}$ is infinite dimensional over $\mathbb{K}$.

Example 3.5. Let $R$ be the same as in Example 3.4, let $R^{\text {op }}$ be the opposite ring, and let $S=R \times R^{\mathrm{op}}$. Then the ring $S$ is semiprimary, but neither left nor right artinian. According to the Hopkins-Levitzki Theorem, the ring $S$ is even neither left nor right noetherian. $S$ has exactly nine $U$-orbits of elements, of principal left ideals and of principal right ideals. Finally, $S$ has an infinite number of $U$-orbits both left and right ideals.

Lemma 3.6. Let $\mathbb{K}$ be an infinite subfield of a field $\mathbb{F}$, and let $R$ be the ring $\left.\left[\begin{array}{c}\mathbb{F} \\ 0\end{array}\right] \mathbb{K}\right]$, by which we mean the ring of matrices of the form $\left[\begin{array}{ll}x & y \\ 0 & z\end{array}\right]$, where $x, y \in \mathbb{F}$ and $z \in \mathbb{K}$, with formal matrix multiplication. Then $R$ has exactly five $U$-orbits both of elements and of principal left (respectively, right) ideals, and exactly six U-orbits of left ideals. Define the classes

$$
\mathcal{K}_{1}=\left\{\left[\begin{array}{ll}
0 & V \\
0 & 0
\end{array}\right] \mid V \subseteq \mathbb{F} \text { is a nonzero } \mathbb{K} \text {-vector subspace }\right\}
$$


and

$$
\begin{array}{r}
\mathcal{K}_{2}=\left\{\mathbb{K} \cdot\left[\begin{array}{ll}
0 & y \\
0 & 1
\end{array}\right] \oplus\left[\begin{array}{ll}
0 & V \\
0 & 0
\end{array}\right] \mid y \in \mathbb{F}\right. \text { and } \\
V \subseteq \mathbb{F} \text { is a nonzero } \mathbb{K} \text {-vector subspace }\}
\end{array}
$$

of right ideals of $R$. Then both the classes $\mathcal{K}_{1}$ and $\mathcal{K}_{2}$ are invariant under the action of the group $U(R) \times U(R)$. There exists a bijection between $U$-orbits in $\mathcal{K}_{1}$ and $U$-orbits in $\mathcal{K}_{2}$.

Proof: From [La91, Proposition 1.17], we can directly check that the ring $R$ has the following right ideals:

1. the zero ideal $0_{2} \cdot R=0_{2}$ and the whole $\operatorname{ring} I_{2} \cdot R=R$;

2. $\left(E_{11}+E_{12}\right) \cdot R=\left[\begin{array}{cc}\mathbb{F} & \mathbb{F} \\ 0 & 0\end{array}\right]$;

3. $\left[\begin{array}{ll}0 & V \\ 0 & 0\end{array}\right]$, where $V$ is a nonzero $\mathbb{K}$-vector subspace of $\mathbb{F}$. $\left[\begin{array}{ll}0 & V \\ 0 & 0\end{array}\right]$ is a principal right ideal of $R$ if and only if $V$ is a 1-dimensional $\mathbb{K}$-vector space;

4. $\left[\begin{array}{ll}0 & y \\ 0 & 1\end{array}\right] \cdot R=\mathbb{K} \cdot\left[\begin{array}{ll}0 & y \\ 0 & 1\end{array}\right]$, where $y \in \mathbb{F}$;

5. $\mathbb{K} \cdot\left[\begin{array}{ll}0 & y \\ 0 & 1\end{array}\right] \oplus\left[\begin{array}{ll}0 & V \\ 0 & 0\end{array}\right]$, where $y \in \mathbb{F}$ and $V$ is a nonzero $\mathbb{K}$-vector subspace of $\mathbb{F}$. None of these is a principal right ideal of $R$.

We leave the determination of (principal) left ideals of the ring $R$ to readers. For the last statement, it is enough to note that for any nonzero $\mathbb{K}$-vector subspaces $V_{1}$ and $V_{2}$ of $\mathbb{F}$, the following statements are equivalent:

1. The right ideals $\left[\begin{array}{cc}0 & V_{1} \\ 0 & 0\end{array}\right]$ and $\left[\begin{array}{cc}0 & V_{2} \\ 0 & 0\end{array}\right]$ of $R$ are $U$-equivalent.

2. The right ideals $\mathbb{K} \cdot\left[\begin{array}{ll}0 & y_{1} \\ 0 & 1\end{array}\right] \oplus\left[\begin{array}{ll}0 & V_{1} \\ 0 & 0\end{array}\right]$ and $\mathbb{K} \cdot\left[\begin{array}{ll}0 & y_{2} \\ 0 & 1\end{array}\right] \oplus\left[\begin{array}{cc}0 & V_{2} \\ 0 & 0\end{array}\right]$ of $R$ are $U$-equivalent for any $y_{1}, y_{2} \in \mathbb{F}$.

3. The right ideals $\mathbb{K} \cdot\left[\begin{array}{ll}0 & y_{1} \\ 0 & 1\end{array}\right] \oplus\left[\begin{array}{cc}0 & V_{1} \\ 0 & 0\end{array}\right]$ and $\mathbb{K} \cdot\left[\begin{array}{cc}0 & y_{2} \\ 0 & 1\end{array}\right] \oplus\left[\begin{array}{cc}0 & V_{2} \\ 0 & 0\end{array}\right]$ of $R$ are $U$-equivalent for some $y_{1}, y_{2} \in \mathbb{F}$.

In particular, a number of orbits under the action of the group $U(R) \times$ $U(R)$ on the class $\mathcal{K}_{1}$ are the same as those on the class $\mathcal{K}_{2}$.

We now apply Lemmas 3.1 and 3.6 to obtain:

Example 3.7. Under the notation of Lemma 3.6, we have:

1. If $[\mathbb{F}: \mathbb{K}]=1$, the ring $R$ has a unique non-principal right ideal, which means that $R$ has exactly six $U$-orbits of right ideals. 
2. If $[\mathbb{F}: \mathbb{K}]=2$ (respectively, $[\mathbb{F}: \mathbb{K}]=3$ ), the ring $R$ has exactly three (five) $U$-orbits of non-principal right ideals, which means that $R$ has exactly eight (ten) $U$-orbits of right ideals.

3. If $[\mathbb{F}: \mathbb{K}] \geq 4$, the ring $R$ has an infinite number of $U$-orbits of right ideals.

Example 3.8. Compare with [Me11, Example 1.5]. Let $\mathbb{F}$ be an infinite field, let $D=\operatorname{diag}(\mathbb{F}, \mathbb{F})$ be the $2 \times 2$ diagonal matrix ring, let $M=$ $M_{2}(\mathbb{F})$ be the $2 \times 2$ matrix ring, and let $R=\left[\begin{array}{cc}D & M \\ 0 & D\end{array}\right]$. According to [La91, Proposition 1.17], ideals of the ring $R$ are of the form $\left[\begin{array}{ll}I & N \\ 0 & J\end{array}\right]$, where $I, J$ are ideals of $D$, and $N$ is a $(D, D)$-subbimodule of $M$ containing $I M+M J$. This means that the ring $R$ has not more than $2^{8}$ ideals and, in consequence, satisfies FNI. Simultaneously, the ring $R$ does not satisfy FNE. For the proof, we let $X_{a}=\left[\begin{array}{cc}a & a^{-1} \\ a & a\end{array}\right]$ for every $a \in \mathbb{F}^{\star}$. Now, it suffices to observe that both matrices $\left[\begin{array}{ccc}0 & X_{a} \\ 0 & 0\end{array}\right]$ and $\left[\begin{array}{cc}0 & X_{b} \\ 0 & 0\end{array}\right]$ belong to the same $U$-orbit if and only if $a= \pm b$. Finally, the ring $R$ is semiperfect as left artinian, and hence does not satisfy FNPLI by [OR03, Lemma 1].

\section{Further comments and questions}

In Theorem 2.1 we saw that for commutative rings, the finiteness of the set of $U$-orbits is closely related to having only a finite number of isomorphism classes of cyclic modules. Now we are going to continue this discussion for arbitrary rings (see for instance [Jo79, JKN88, OR03]).

Theorem 4.1. For every ring $R$, the following statements are equivalent:

1. $R$ satisfies FNLI.

2. $R$ is semilocal and has only a finite number of isomorphism classes of cyclic modules.

3. $R$ is semilocal and has only a finite number of isomorphism classes of indecomposable cyclic modules.

Proof: $1 \Rightarrow 2$. Due to Theorem 2.3, the $\operatorname{ring} R$ is semilocal.

We know that every cyclic $R$-module is isomorphic to the quotient $R$-module $R / I$ for some left ideal $I$ of $R$, and that if left ideals $I$ and $J$ of $R$ belong to the same $U$-orbit, then the $R$-modules $R / I$ and $R / J$ are isomorphic. Since $R$ satisfies FNLI, we see that $R$ has only a finite number of isomorphism classes of cyclic modules. 
The implication $2 \Rightarrow 1$ follows easily from [OR03, Corollary 3], while the implication $2 \Rightarrow 3$ is obvious.

$3 \Rightarrow 2$. For an $R$-module $M$, we denote by $J(M)$ the Jacobson radical of $M$. Let $\bar{M}=M / J(M)$ and $\bar{R}=R / J(R)$. Since, by assumption, $R$ is semilocal, hence $J(M)=J(R) M$, and, in particular, $\bar{M}$ is a semisimple $\bar{R}$-module (see [La91, Proposition 24.4] for more details). If $M$ is a cyclic $R$-module, then $\bar{M}$ is an $\bar{R}$-module of finite length, not greater than this of $\bar{R}$. Moreover, if $M$ is a cyclic $R$-module with $\bar{M}$ simple, then $M$ is indecomposable.

Now, by assumption, $R$ has only a finite number of isomorphism classes of indecomposable cyclic modules. By induction on the length of $\bar{M}$, we can check that there are only a finite number of isomorphism classes of all cyclic $R$-modules.

The so-called Cozzens domains demonstrate that the last two of statements in Theorem 4.1 are not equivalent if the ring is not semilocal. For more details on Cozzens domains we refer the readers to [Fa81].

Theorem 4.1 combined with Theorems 2.3 and 2.7 asserts that if a ring $R$ is left fully bounded and every prime quotient ring of $R$ is left Goldie, then the condition of having only a finite number of indecomposable cyclic modules forces $R$ to be left artinian. This result has previously been proved by Josef Stock [Sto86]. His technique differs from that used by us.

Corollary 4.2. For any ring $R$ and any positive integer $n$, the $n \times n$ matrix ring $M_{n}(R)$ satisfies FNLI if and only if $R$ is semilocal and has only a finite number of isomorphism classes of modules with at most $n$ generators. In particular, if $M_{n}(R)$ satisfies FNLI, then $M_{m}(R)$ satisfies FNLI for every positive integer $m \leq n$.

Proof: We know that a ring $R$ is semilocal if and only if the matrix ring $M_{n}(R)$ is semilocal (see for instance [La91, Example 20.4]). Moreover, as in the proof of [OR03, Theorem 7], we have a correspondence between isomorphism classes of $R$-modules generated by at most $n$ elements and isomorphism classes of cyclic $M_{n}(R)$-modules. The corollary now follows directly from Theorem 4.1.

For a ring $R$, the action (1.1) induces in a natural way an action of the group $U(R) \times U(R)$ on the set of all subgroups of the additive group $R^{+}$. About rings having only a finite number of $U$-orbits of subgroups of $R^{+}$ we have: 
Proposition 4.3. If a ring $R$ has only a finite number of $U$-orbits of subgroups of $R^{+}$, then $R$ is finite.

Proof: Evidently, the ring $R$ satisfies FNI. Due to Theorem 2.2, $R=$ $S \oplus T$ where $S$ is a $\mathbb{Q}$-algebra and $m T=0$ for some $m \geq 1$.

Suppose that there exists a nonzero element $x \in S$. Then for every prime number $p$, we can take the subgroup $A_{p}=\mathbb{Z}[1 / p] x$, where $\mathbb{Z}[1 / p]=\sum_{n>1} \mathbb{Z} p^{-n}$. It is obvious, by $p$-divisibility argument, that the subgroups $A_{p}^{-}$belong to different $U$-orbits, a contradiction. This means that $S=0$, and so $R=T$.

If $R$ is an infinite ring, then $R$ has an infinite number of subgroups of different orders which belong to different $U$-orbits, again a contradiction. It follows that $R$ is a finite ring, as required.

Under the assumption on the finiteness of the set of $U$-orbits of cyclic subgroups of $R^{+}$, we can only prove:

Proposition 4.4. If a ring $R$ has only a finite number of $U$-orbits of cyclic subgroups of $R^{+}$, then $R=S \oplus T$, where $S$ is an algebra over the field $\mathbb{Q}$, and $m T=0$ for some positive integer $m$. Any of these summands can be infinite.

Proof: By assumption, elements of the group $R^{+}$have only a finite number of orders. This means that $m T=0$ for the torsion part $T$ of $R^{+}$and some $m \geq 1$. Let $\bar{R}=R / T$. For a prime number $p$, we suppose that an element $x \in \bar{R}$ is not divisible by $p$. Then for every $n \geq 1$ the element $p^{n} x$ is divisible by $p^{n}$ but not by $p^{n+1}$. Thus we have constructed an infinite number of elements generating cyclic subgroups of $R^{+}$belonging to different $U$-orbits, a contradiction. It follows that $\bar{R}^{+}$is a divisible group. Now, as in the proof of the statement 2 in Theorem 2.2, we obtain $R=S \oplus T$, where $S \cong \bar{R}$ as a $\mathbb{Q}$-algebra.

Finally, if $\mathbb{K}$ is a field of any characteristic, then $\mathbb{K}^{+}$has exactly two $U$-orbits of cyclic subgroups, and the result follows.

The connections (1.2) hold if we replace (principal) left ideals by (principal) right ideals. In this case the properties FNE and FNI are still the same, while the properties FNLI and FNPLI are replaced by FNRI and FNPRI, respectively. Formula (1.2) and its right-side version state that

$$
\mathrm{FNE} \Rightarrow \mathrm{FNPLI} \wedge \mathrm{FNPRI} \text {. }
$$

We may ask whether the converse implication is true. For another formulation of this question, let us recall that a subgroup $Q$ of $R^{+}$, in our unital ring $R$ is a quasi-ideal if $Q$ is an intersection of a left and a right 
ideal of $R$ (see [Ste78, §2] for elementary properties of quasi-ideals). A quasi-ideal $Q$ of the ring $R$ is principal if $Q$ is an intersection of a principal left and a principal right ideal of $R$. Obviously, quasi-ideals of $R$ are invariant under the action (1.1). If we define the properties FNQ and FNPQ in a natural way, then our question is: Does the property FNPQ imply the property FNE? Another question in this area is: Must every ring satisfying $F N Q$ be semiprimary? From our examples we know that the property FNQ is stronger than the property FNLI. On the other hand we know that such rings must be semilocal.

Rings satisfying properties discussed here should have many units. For a ring $R$ and a positive integer $n$, let $U_{n}(R)$ denote the set of all sums of no more than $n$ units, and let $R_{U}=\sum_{n \geq 1} U_{n}(R)$. Such subsets are frequently considered in the literature. For more details, see [Sr10] and the references given there. In every ring $R$ the subset $R_{U}$ is a subring invariant under the action (1.1). If $R$ satisfies FNE then $R_{U}=U_{n}(R)$ for any sufficiently large $n$ and $R$ is a finitely generated $\left(R_{U}, R_{U}\right)$-bimodule. The converse implication is not true even if $R$ satisfies FNI. If, for example, $\mathbb{K}$ is a countable field, and $A$ is a simple nil-algebra over $\mathbb{K}$ constructed by Agata Smoktunowicz in [Sm02], then we can take $R$ as the extension of $A$ to a unital algebra with the help of $\mathbb{K}$. It is easy to see that $R=U_{2}(R)$ but $R$ does not satisfy FNE and even FNPLI, by Lemma 2.5. There are also $J$-semisimple examples of this kind.

Example 4.5. Let $D$ be a division ring. For any non-negative integer $n$, let $R_{n}$ denote the $2^{n} \times 2^{n}$ matrix ring $M_{2^{n}}(D)$. We can regard $R_{n}$ as a subring of $R_{n+1}$ by identifying a $2^{n} \times 2^{n}$ matrix $X$ with the $2^{n+1} \times$ $2^{n+1}$ matrix $\left[\begin{array}{cc}X & 0 \\ 0 & X\end{array}\right]$. Let $R$ be the union $\bigcup_{n \geq 0} R_{n}$. According to [La91, pp. 42-43], $R$ is a simple and $J$-semisimple non-artinian ring. We can check, as in [Sr10], that $R=U_{2}(R)$ but $R$ does not satisfy FNPLI.

In connection with Corollary 4.2 and some results from [OR03] and [Me11], we may ask about Morita equivalence of the finiteness of the set of $U$-orbits of elements, of (principal) left ideals, of quasi-ideals, and of other $U$-invariant subsets of rings. Some interesting results in this area are recently obtained by Arkadiusz Męcel and Jan Okniński in [MO13] for finite dimensional algebras over fields.

\section{References}

[Fa81] C. FAITH, "Algebra. I. Rings, modules, and categories", Corrected reprint, Grundlehren der Mathematischen Wissenschaften 190, Springer-Verlag, Berlin-New York, 1981. 
[Fu73] L. Fuchs, "Infinite abelian groups", Vol. II, Pure and Applied Mathematics 36-II, Academic Press, New York-London, 1973.

[Hi04] Y. HiRAno, Rings with finitely many orbits under the regular action, in: "Rings, modules, algebras, and abelian groups", Lecture Notes in Pure and Appl. Math. 236, Dekker, New York, 2004, pp. 343-347.

[Jo79] S. JøNDRUP, Indecomposable modules, in: "Ring theory" (Proc. Antwerp Conf. (NATO Adv. Study Inst.), Univ. Antwerp, Antwerp, 1978), Lecture Notes in Pure and Appl. Math. 51, Dekker, New York, 1979, pp. 97-104.

[JKN88] S. Jøndrup, J. Krempa, And D. Niewieczerzą, A note on indecomposable modules, Rend. Circ. Mat. Palermo (2) 37(1) (1988), 100-108. DOI: $10.1007 / \mathrm{BF} 02844270$.

[La91] T. Y. LAM, "A first course in noncommutative rings", Graduate Texts in Mathematics 131, Springer-Verlag, New York, 1991. DOI : $10.1007 / 978-1-4684-0406-7$.

[Me11] A. MȨCEL, Algebras with finitely many orbits with respect to the unit group, Preprint, Institute of Mathematics, University of Warsaw.

[MO13] A. MȩCEL AND J. OKniŃsKi, Conjugacy classes of left ideals of a finite dimensional algebra, Publ. Mat. 57(2) (2013), 477-496. DOI : $10.5565 /$ PUBLMAT_57213_10.

[Ok98] J. OkNIŃsKI, "Semigroups of matrices", Series in Algebra 6, World Scientific Publishing Co., Inc., River Edge, NJ, 1998. DOI : $10.1142 / 9789812816290$.

[OR03] J. OKniŃski AND L. E. RenneR, Algebras with finitely many orbits, J. Algebra 264(2) (2003), 479-495. DOI: 10.1016/ S0021-8693(03) 00129-7.

[Sm02] A. Smoktunowicz, A simple nil ring exists, Comm. Algebra 30(1) (2002), 27-59. DOI : 10.1081/AGB-120006478.

[Sr10] A. K. Srivastava, A survey of rings generated by units, Ann. Fac. Sci. Toulouse Math. (6) 19 (2010), Fascicule Spécial, 203-213.

[Ste78] O. Steinfeld, "Quasi-ideals in rings and semigroups", With a foreword by L. Rédei, Disquisitiones Mathematicae Hungaricae 10, Akadémiai Kiadó, Budapest, 1978.

[Sto86] J. STосK, A note on rings having only a finite number of cyclic indecomposable modules, Arch. Math. (Basel) 47(5) (1986), 408-409. DOI : $10.1007 /$ BF01189979. 
Małgorzata Hryniewicka:

Institute of Mathematics

University of Białystok

Akademicka 2

15-267 Białystok

Poland

E-mail address: margitt@math.uwb.edu.pl

Jan Krempa:

Institute of Mathematics

University of Warsaw

Banacha 2

02-097 Warszawa

Poland

E-mail address: jkrempa@mimuw.edu.pl

Primera versió rebuda el 20 de desembre de 2012, darrera versió rebuda el 29 d'abril de 2013. 